\title{
The Application of Dietary Fiber in Bread Products
}

Marcin Kurek* and Jarosław Wyrwisz

Division of Engineering in Nutrition, Faculty of Human Nutrition and Consumer Sciences, Warsaw University of Life Sciences, Poland

\begin{abstract}
The process involved in the manufacture of bread is highly complex to ensure production of high quality bread, and a number of ingredients are incorporated in the process. Increased consumer health awareness has triggered interest in adding functional ingredients, such as dietary fiber (DF), in popular foods like bread. The article reviews the in the function of DF in human diet and most important sources of DF used in the bread productions. The impact was put on the effect of DF incorporation in bread on rheology of dough and properties of final product. The review revealed the dependence of DF usage in bread production in terms of changing the physical parameters of bread, shelf-life and sensory characteristics of bread.
\end{abstract}

Keywords: Bread; Dietary fiber; Read making; Shelf-life; Rheological parameters; Dough

\section{Introduction}

Dietary fiber is currently considered a critical ingredient in food products. The popularity of DF in food products is caused by an increased interest in developing health foods, and popular food products, particularly bread, are currently being used as vehicles for DF. Dietary fiber is a mixture of plant carbohydrate polymers, consisting of polysaccharides and oligosaccharides, as well as hemicelluloses, cellulose, resistant starch, pectin substances, inulin, and gums, among others [1]. In addition to evading digestion, absorption, and hydrolysis, DF is also involved in increasing fecal bulk, stimulating colonic fermentation, and reducing pre-prandial cholesterol levels and levels of postprandial blood glucose in the body of the organism [2]. The application of DF in bread making is also occasioned by its technological properties. For example, the water-holding capacity of DF prevents the bread from staling, which could extend the shelf-life of the product. This study reviews the importance of DF in the human diet, major sources of DF in bread making, effects of DF on rheological parameters of dough, and effects of DF on shelf-life of bread among other important areas to be covered in this study.

\section{The Importance of Dietary Fiber in the Human Diet}

Studies show that DF plays a pivotal role in the human diet. According to epidemiological studies, increased consumption of DF content significantly reduces obesity. Such findings have also been emphasized by Slavin [3] who argues that available epidemiological studies indicate that DF intake lowers the risk of individuals developing obesity and certain kinds of cancer. High content of DF in the diet provides a plethora of health benefits, including prevention of diabetes, heart disease, certain cancers, and weight gain. In addition, diary DF can also enhance digestive health.

According to Angioloni and Collar [4], a number of health benefits are linked to dietary DF, among them promoting healthier bowel function, decreasing cholesterol levels in the body, and controlling blood glucose levels. DF increases the volume of stools as well as softening them, thereby preventing constipation and, at the same time, maintaining bowel health.

Moreover, insoluble DF lowers transit time, limiting how long harmful substances could come into contact with the intestinal walls. In addition to promoting healthier bowel function, DF also plays a crucial role in regulating blood sugar levels. Soluble DF contributes to slowing down the release of sugar from the digested food into the bloodstream [5]. This indirectly helps to prevent rapid rises in blood sugar levels which often result in the development type of 2 diabetes. Other important roles of DF in the human diet include reduction of cholesterol levels in the body, as well as regulating weight gain [6].

\section{Main Sources of DF in Bread Making}

Bread is a staple food, which is basically prepared by making dough out of water and flour. Various types of flour and ingredients are used in a number of combinations and proportions in the preparation of bread. Additionally, there are different recipes and modes of preparing bread. Because of this, bread comes in a variety of sizes, shapes, and textures. Bread is a leavened food normally produced through fermentation of sugars that have been obtained from starch (commonly wheat flour), and which results in chemical interactions between a number of components in the food. Varied ingredients are used during the bread making process for the purpose of ensuring the development of the continuous protein network that is indispensable in guaranteeing the quality of bread [7].

Angioloni and Collar [4] showed that bread making calls for a careful selection of DF, choosing fiber with the most appropriate physicochemical properties to prevent permanent protein matrix disruption for purposes of avoiding excessive weakening, particularly in highly substituted flour systems. In an attempt to come up with a low-calorie bread, a multi-DF strategy has been applied [8]. In addition to wheat which contains a mixture of soluble and insoluble pentosans in endosperm and the insoluble hemicelluloses available in bran, other sources of DF used in the production of bread included rice bran, soy DF, corn bran, powdered cellulose, and oat DF. The criteria used in selecting the DF to be used include color, DF content, cost, and water absorption capacity, along with water retention capacity following baking. Reducing the particle size of the fiber powder allows for an increase of water-holding capacity by increasing the degree of surface development. In the bread production process, this feature

*Corresonding author: Marcin Kurek, Division of Engineering in Nutrition, Faculty of Human Nutrition and Consumer Sciences, Warsaw University of Life Sciences, 02-776 Warsaw, Poland, Tel: +48 2259037 014; E-mail: marcin_kurek@sggw.pl

Received March 14, 2015; Accepted April 08, 2015; Published April 15, 2015

Citation: Kurek M, Wyrwisz J (2015) The Application of Dietary Fiber in Bread Products. J Food Process Technol 6: 447. doi:10.4172/2157-7110.1000447

Copyright: @ 2015 Kurek M, et al. This is an open-access article distributed under the terms of the Creative Commons Attribution License, which permits unrestricted use, distribution, and reproduction in any medium, provided the original author and source are credited. 
is extremely important, because water is involved in the processes of starch gelatinization, protein denaturation, and formation of flavor characteristics. It also significantly affects the color. Moreover, water content influenced the staling kinetics in bread $[9,10]$.

\section{Effect of Dietary Fiber on Rheological Parameters of Dough}

Basically, the process involved in the production of bread is a highly complex one that entails a range of parameters that must be regulated. According to Almeida et al. [11], rheological measurements are used in the prediction of both the behavior of wheat dough during the bread production process and of the quality of the final product. Despite the fact that there are different sources of DF, Almeida et al. [11] argue that DF used in bread production affects both processing and final product quality.

The effects of DF on rheological parameters of dough has been extensively explored and documented in the literature. For instance, a study completed by Gomez et al. [2] sought to discover the effects of adding various types of DF on the rheological properties of wheat dough. They found that the larger the dough elongation, the looser the dough. Furthermore, this study found that values that are too low are undesirable in the production of bread with the required parameters.

According to Rosell et al. [9], the incorporation of DF into the bread dough system significantly impedes protein association, and influences bread behavior during the cooling and heating processes. For instance, incorporation of sugar beet DF in the dough matrix stimulates disruption of the viscoelastic system, resulting in the production of weaker dough. Moreover, it competes for water with the available starch hence influencing gelling and pasting.

The study done by Peressini and Sensidoni [12] also sought to determine the effect of inulin addition on the rheological properties of common wheat dough and on the quality of the resultant bread. During the study, three commercial fructan products were used, which were of a varied number of average degrees of polymerization. In this experimental study, the rheological properties of the dough were examined using dynamic rheological and farinographical measurements. The findings of the study showed that the addition of DF led to a considerable increase in stability as well as mixing time. Furthermore, the experiment also recorded a decrease in water absorption.

A study done by Saeed et al. [13] also investigated the impact of DF on the rheological parameters of dough. In order to determine impact, $2-20 \%$ rice bran was added to wheat flour and the rheological parameters of the dough examined using a consistograph, a farinograph, and an alveograph. This study noted insignificant changes in the physicochemical properties of the dough. This was noted even after the addition of $20 \%$ bran. Based on the findings of the study, it was crystal clear that adding hydrophobic rice bran to wheat had a negative impact on the mixture, leading to inferior performance in dough development and lower water absorption capacity [14].

\section{Effect of dietary fiber on physical parameters of bread}

A number of studies in the literature, both past and present, have extensively explored the effects of DF on physical parameters of bread. In this section, the effects of DF on volume, yield, moisture loss, color, and texture will be widely explored. The yield, volume and moisture loss are the most important factors while considering the technological quality of bread due to economic reasons. The quality of the product could be assessed by the color, texture, shelf life and sensory properties. Thus, the above parameters are discussed in the paragraph.

\section{Yield, volume, moisture loss}

Baking is the most important step in the bread production which transforms the raw dough to the bread using the heating. During the baking the weight loss is observed due to losing the water which was incorporated into the dough during mixing process. As already documented, DF may have a negative effect on the final bread quality leading to reduced volume of the bakery product, i.e. loaf volume $[15,16]$. In the study done by Anil [17], the findings included reduction in bread volume following the addition of hazelnut DF. The study by Almeida et al. [11] found that when DF was introduced into bread, their specific volume coupled with crumb luminosity reduced significantly.

In the study done by Rubel et al. [15], it was found that DF considerably decreased loaf volume beyond what would be expected from gluten dilution. The significant decrease in loaf volume was attributed to lower gas retention as opposed to unsatisfactory production of gas. Furthermore, the effects of the addition of DF on texture and color were also determined. The overall effect of adding DF from cellulose was smaller compared to bran. For instance, bran DF resulted in a decrease in bread softness to a much higher degree than did cellulose. Based on the literature reviewed above, it is confirmed that DF always tends to lead to a reduction in volume and moisture loss [18].

Oat is known as a DF source with high protein value, compared to other cereals used in the process of making bread. Oat flour used in bread making should result in increased biological value of the product. Bread quality was analyzed in terms of volume and total loss during baking showing the lowest total loss in the samples with oat fiber used in bread production [19].

\section{Texture}

The DF in dietary products has been known to alter their texture and consistency. DF can modify the firmness and springiness of the bread but these modifications are mainly affected by the fiber source. The impact on texture has also been studied by Amir et al. [20], who argued that incorporating DF from cocoa pod husk powder (CPHP) had a considerable effect on both firmness and volume of the bread. During the study, researchers realized that adding CPHP made the bread denser and firmerr in texture [21]. Conversely, other studies have taken note of the fact that dietary DF can result in improved texture. DF has been found to improve stability and strength of the final product. In fine bread, soluble DF such as polydextrose has been found to interfere with the formation of gluten; hence the creation of a short texture for products such as cookies and biscuits, while in chocolate-covered bread, polydextrose can be used along with polyols to improve texture and taste.

Retro gradation of amylose and amylopectin is a process that leads to staling of bread. For this reason, more and more interest has been paid to producing complex compounds with amylopectin and amylose in order to inhibit retro gradation, e.g. with substances belonging to dietary fiber groups. Using high amounts of insoluble fiber in bread caused increased product firmness during storage. Both decreased firmness and decreased volume are affected by the content of dietary fiber in the used bread. When the product is heated and water is added, hydrocolloids with strong bonds are formed, and these bonds cannot be broken by enzymes in the flour due to a low amount of enzymes. One of such example of such mechanism is the incorporation of $\beta$-glucan in 
bread made from wheat flour with small amount of $\beta$-glucanase [22]. Smaller changes in firmness value were observed for fiber with larger particles, and there was less discrepancy with fiber particles that were larger than a control sample. Due to its water binding capacities, fiber inhibits water loss during storage and prevents staling that affects crumb firmness [23]. The fact that firmness is impaired with increased fiber content may be associated with DF exhibiting increased water capacity, so water does not become available for gluten that is formed while kneading [24]. According to another theory, the physical properties of bread can be impaired due to reduced fiber particle size, as the surface is developed to a higher degree, wherefore chemical reactions can occur more easily, affecting the physical parameters [25]. Fiber micronization may also be associated with the fact that the aleuronic layer of cells is destroyed while grinding, resulting in the release of enzymes, glutathione, or phytates, which may impair gluten formation due to chemical reactions Chen et al. [26].

\section{Color}

Golden brown crust and creamy white bread crumb are the most important appealing factors to indicate the quality of a bakery product to consumers.

It has been observed that crust tends to be browner with a higher content of DF, as the crust of wheat rolls with high dietary fiber underwent a color change due to an oxidation reaction and sugars participated in caramelization during baking [27]. The DF content could influence the amount of the produced melanoids which are one of the most important indicators of Maillard reactions [28]. DF could change the However, the caramelization process and the Maillard reaction is not the only causes for color change in bread with added DF. This is true only when we want to explain the surface color of bread that has been directly exposed to high temperatures. In the case of bread crumb, such high temperatures are not achieved; therefore it is reasonable to perform measurements only for crumb, the color of which directly correlates with the ingredients used for dough production. Results obtained in this study indicate that a lower size of DF added to bread results in lower differences compared to a control sample. However, it is worth indicating that reduced lightness of bread may be a result of water being bound by fiber, as it is a milieu of reactions between sugars and amino acids [29].

\section{Effect of DF on Shelf-Life of Bread}

Bread is generally a perishable product with a shelf-life that is limited by the physicochemical deterioration known as staling, which leads to hardening and a dry texture connected with losing the moisture of bread [30]. DF could play important role in extending the shelf life considering quality issues. There were some researches that were conducted regarding this issue. DF, plays several roles including increasing the shelf-life of foods. This is made possible because of certain characteristics, such as gel-forming, water-binding, antisticking, fat mimicking, rising, and thickening capacities.

The application of DF in the bread making process leads to enhancement of oil and water holding capacities, reduction of syneresis, modification of textural properties, and, above all, it improves the shelf-life of bread. This concept was further confirmed by Sabanis et al. [10] who asserted that enhancing shelf-life is among the functional properties of the DF, especially when included in food products, such as bread. Moreover, Penella et al. [23] assert that a number of recent studies show that DF can act as an appropriate viscoelastic profile for the formulation of bread dough, likely giving the final product a favorable shelf-life.
Furthermore, the importance of DF in improving the shelf-life of bread was examined in a study geared towards developing a means for the optimization of the biochemical activity of sourdough to attain improved bread texture, flavor, and shelf-life, as well as towards determining how the aging process of fresh bread is altered using sourdough. The study found that the addition of DF tremendously improved the shelf-life of bread. The use of sourdough when baking with wheat has been reported to have mixed effects on the shelf-life of wheat bread.

\section{Effect of DF on sensory characteristics of bread}

A number of researchers have successfully used wheat bran for purposes of enhancing the nutritional quality of baked products, such as cakes, muffins, cookies, and yeast breads. According to Yangilar [5], addition of wheat bran has tremendous effect on the physical as well as the sensory properties associated with baked products. The study done by Grossi et al. [29] sought to determine the synergy between high pressure treatment and carrot DF. In this experiment, sensory evaluation showed that the high pressure treatment worked synergistically with the carrot DF to enhance sensorial attributes, such as creaminess, homogeneity, and firmness.

According to Almeida et al. [30], in regards to the sensory analysis done during the study, the DF sources studied had a considerable influence on acceptance of color appearance, crumb color, and texture. The results of the study done by Feili [29] showed that incorporation of DF in varied ratios led to considerable influence on texture, as well as bread volume attributes.

In addition, Gómez et al. [2] posited that addition of DF to bread dough not only extends the shelf-life of the product, but also significantly enhances both qualitative and sensory properties of bread. According to Almeida et al. [30], based on the sensory analysis done, the DF studied had a significant effect on color, crumb acceptability, and texture.

\section{Conclusion}

Based on available studies, it is crystal clear that DF is a significant component of diet and nutrition. In addition, a food group, DF is considered to hold extreme biological significance, in spite of being insufficiently digested. Studies have analyzed a number of health benefits associated with dietary DF, including promotion of healthier bowel function, lowering the level of cholesterol in the body, and controlling blood sugar levels. The study has also examined the major sources of DF used in bread making. The reviewed literature showed that DF plays a critical role in the shelf-life of dietary products. Other important areas discussed included the effects of the DF on rheological parameters of dough, on physical parameters of bread, shelf-life of bread, and on sensory characteristics of bread. The health benefits and positive effects on shelf-life provided by dietary fiber are significant and justify substantial further research into developing methods for limiting the perceived negative effects of fiber on aspects such as texture, color, and volume.

\section{Acknowledgement}

The study has been conducted within the internal grant of Warsaw University of Life Sciences number: 505-10-102800-L00377-99

\section{References}

1. Fuentes-Zaragoza E, Riquelme-Navarrete MJ, Sanchez-Zapata E, PerezAlvarez JA (2010) Resistant starch as functional ingredient: a review. Food Research International 43: 931-942. 
Citation: Kurek M, Wyrwisz J (2015) The Application of Dietary Fiber in Bread Products. J Food Process Technol 6: 447. doi:10.4172/21577110.1000447

2. Gomez M, Moraleja A, Oliete B, Ruiz E, Caballero PA (2010) Effect of fibre size on the quality of fibre-enriched layer cake. LWT-Food Science and Technology 43: $33-38$

3. Slavin J (2005) Dietary DF and body weight. Nutrition 21: 411-418.

4. Angioloni A, Collar C (2011) Physicochemical and nutritional properties of reduced caloric density high-fibre breads. LWT-Food Science and Technology 44: 747-758

5. Yangilar F (2013) The application of dietary fibre in food industry: structural features, effects on health and definition, obtaining and analysis of dietary fibre: a review. Journal of Food and Nutrition Research 1: 13-23.

6. Devi S, Cheong J, Kim I (2014) Effects of dietary fiber and benzoic acid on growth performance, nutrient digestibility, reduction of harmful gases, and lipid profiles in growing pigs. Annals of Animal Science, South Korea.

7. Sivam AS, Sun-Waterhouse D, Quek SY, Perera CO (2010) Properties of bread dough with added fibre polysaccharides and phenolic antioxidants: A review. Journal of Food Science 75: 163-174.

8. Collar C, Santos E, Rosell M (2006) Significance of DF on the viscometric pattern of pasted and gelled flour-fibre blends. Cereal Chemistry 84: 370-376.

9. Rosell C, Santos E, Collar C (2010) Physical characterization of fiber-enriched bread doughs by dual mixing and temperature constraint using the Mixolab. European Food Research and Technology 231: 499-634.

10. Sabanis D, Lebesi D, Tzia C (2009) Effect of dietary fibre enrichment on selected properties of gluten-free bread. LWT-Food Science Technology 142: 1380-1389.

11. Almeida E, Chang Y, Steel C (2010) Effect of adding different dietary DF sources on farinographic parameters of wheat flour. Cereal Chemistry 87: 566-573.

12. Peressini D, Sensidoni A (2009) Effect of soluble dietary fibre addition on rheological and bread making properties of wheat dough. Journal of Cereal Science 49: 190-201

13. Saeed E (2009) Influence of rice bran on rheological properties of dough and in the new product development. Journal of Food Science Technology 46: 62-65.

14. Mohamed S (2014) Functional food against metabolic syndrome (obesity, diabetes, hypertension and dislipidemia) and cardiovascular diseases. Trends in Food Science and Technology 35: 114-128.

15. Rubel IA, Perez EE, Manrique GD, Genovese DB (2015) Fibre enrichment of wheat bread with Jerusalem artichoke inulin: Effect on dough rheology and bread quality. Food Structure 3: 21-29.
16. Khater GE, Bahnasawy A (2014) Heat and mass balance for baking process. Bioprocessing and Biotechniques 4: 1-6.

17. Anil M (2007) Using of hazelnut testa as a source of dietary DF in breadmaking Journal of Food Engineering 80: 61-67.

18. Dhingra D (2012) Dietary fibre in foods: a review. Journal of Food Science Technolnology 49: 255-266.

19. Lenka D, Polakovicova P, Rakicka M, Sturdik E (2013) Characterization and selection of cereals for preparation and utilization of fermented fiber-betaglucan product. Journal of Microbiology and Food Sciences 2: 1384-1404.

20. Amir I, Hanida H, Syafiq A (2013) Development and physical analysis of high DF bread incorporated with cocoa (Theobroma cacaosp.) pod husk powder. International Food Research Journal 20: 1301-1305.

21. Alemawor F, Dzogbefia, V, Oddoye E (2009) Enzyme cocktail for enhancing poultry utilization of cocoa pod husk. Academic Journals 4: 555-559.

22. Elleuch E, Bedigian D, Roiseux O, Besbes S, Blecker C, et al. (2011) Dietary fibre and fibre-rich by products of food processing: characterisation, technological functionality and commercial applications: a review. Food Chemistry 124: 411-421.

23. Walter T, Wieser H, Koehler P (2014) Degradation of gluten in wheat bran and bread drink by means of a proline-specific peptidase. Journal of Nutrition \& Food Sciences 4: 1-6.

24. Ranasalva N, Visvanathan R (2014) Development of bread from fermented pearl millet flour. Journal of Food Processing and Technology 5: 1-5.

25. Sathivel S, Ram AK, Espinoza L, King J, Cuetov R, et al. (2013) Application of Honey Powder in Bread and its Effect on Bread Characteristics. Journal of Food Processing and Technology 4: 1-6.

26. Chen J, Gao D, Yang L, Gao Y (2013) Effect of microfluidization process on the functional properties of insoluble dietary fiber. Food Research International 54: $1821-1827$

27. Grossi E (2011) Synergistic cooperation of high pressure and carrot dietary fibre on texture and colour of pork sausages. Meat Science 89: 195-201.

28. Almeida E, Chang Y, Steel C (2012) Dietary fibre sources in bread: Influence on technological quality. LWT- Food Science and Technology 50: 545-553.

29. Feili R (2013) Physical and sensory analysis of high dietary fiber bread incorporated with jackfruit rind flour. Food Science and Technology 1: 30-36.

30. Peighambardoust SH, Aghamirzaei M (2014) Physicochemical, nutritional, shelf life and sensory properties of iranian sangak bread fortified with grape seed powder. Journal of Food Processing and Technology 5: 1-6. 